operability. Furthermore, the preservation of hepatic parenchyma by NAR may enable a larger proportion of patients subsequently developing recurrent metastatic disease to undergo repeat metastasectomy.

Competing interests None declared.

\section{PTU-075 LIVER DYSFUNCTION AFTER MAJOR HEPATIC RESECTION}

doi:10.1136/gutjnl-2012-302514c.75

${ }^{1,2} \mathrm{D}$ C Bartlett, ${ }^{2} \mathrm{E}$ L Neo, ${ }^{2} \mathrm{G}$ Bonney, ${ }^{2} \mathrm{R}$ Marudanayagam, ${ }^{2} \mathrm{D}$ Mirza. ${ }^{1} \mathrm{~N} / \mathrm{HR}$ Biomedical Unit and Centre For Liver Research, University of Birmingham, Birmingham, UK; ${ }^{2}$ Liver Unit, Queen Elizabeth Hospital, Birmingham, UK

Introduction Liver failure is a potentially fatal complication following major hepatic resection. This study evaluates the incidence and factors associated with posthepatectomy liver failure (PHLF), as well as the outcomes and survival of these patients.

Methods All patients who underwent elective major hepatic resection (>4 liver segments) between January 2001 and March 2011 were identified from a prospective database. Patients with bilirubin levels $>100 \mathrm{mmol} / \mathrm{l}$ or INR $>2$ on three consecutive days within the first post-operative week were diagnosed with PHLF $(n=54)$. These patients were compared with 654 control patients.

Results Patients with PHLF had a higher incidence of diabetes mellitus compared to the control group ( $9.5 \%$ vs $3.1 \%, \mathrm{p}=0.05$ ). There was no significant difference in age, pre-operative chemotherapy, weight of resected specimen, use of Pringle manoeuvre, degree of steatosis/fibrosis of background liver, or amount of perioperative blood transfusion. Post-operatively, patients with PHLF were more likely to require n-acetylcysteine $(51.4 \%$ vs $13.4 \%$, $\mathrm{p}<0.001)$ and dialysis $(13.2 \%$ vs $1.8 \%, \mathrm{p}<0.001)$, and had longer ITU stay (mean 2.57 days vs 0.84 days, $\mathrm{p}<0.001$ ). The PHLF group had higher 30 -day $(22.6 \%$ vs $3.1 \%, \mathrm{p}<0.001)$ and 90 -day mortality $(41.5 \%$ vs $4.7 \%, p<0.001)$. Median survival was 9.86 months in the PHLF group and 49.77 months in the control group ( $p<0.001)$.

Conclusion This study over a 10 -year period has shown a small risk of PHLF (7.6\%) in patients undergoing major hepatic resection. PHLF is associated with significantly increased post-operative morbidity and mortality.

Competing interests None declared.

\section{PTU-076 FACTORS AT FIRST ERCP WHICH INFLUENCE THE DECISION TO PROCEED TO SUBSEQUENT SURGERY OR REPEAT ERCP FOR COMPLEX BILIARY PROBLEMS}

doi:10.1136/gutjnl-2012-302514c.76

${ }^{1} \mathrm{E}$ Watts, ${ }^{*}{ }^{2} \mathrm{H}$ Nair, ${ }^{2} \mathrm{~S}$ Bow, ${ }^{2} \mathrm{~A}$ MacGilchrist, ${ }^{2} \mathrm{~A}$ Bathgate, ${ }^{2} \mathrm{~J}$ Plevris, ${ }^{2} \mathrm{~J}$ Casey, ${ }^{2} \mathrm{~J}$ Powell, ${ }^{1} \mathrm{~J}$ Garden, ${ }^{1} \mathrm{R}$ Parks, ${ }^{1} \mathrm{M}$ Duxbury, ${ }^{2} \mathrm{~N}$ Church, ${ }^{1} \mathrm{D}$ Mole, ${ }^{2} \mathrm{R}$ Ravindran. ${ }^{1}$ University of Edinburgh, Edinburgh, UK; ${ }^{2}$ Royal Infirmary, Edinburgh, UK

Introduction ERCP is a safe and highly effective solution to many pancreaticobiliary problems. However, surgical options also exist. After a challenging first ERCP, it can be unclear whether surgery or repeat ERCP is preferred. The aim of this study was to identify predictive factors at first ERCP which inform this decision.

Methods All ERCPs performed at one hospital (April 2008-March 2011) were analysed. Patients having more than one ERCP were evaluated in detail. Demographics, disease-specific and procedurespecific variables relating to ERCPs and any subsequent surgery were extracted. The primary outcome measure was a requirement for surgery after two or more ERCPs. Descriptive statistics and logistic regression were performed.
Results 1729 ERCPs were done in 1270 patients, of which 317 patients had more than one ERCP. Of these, 140 patients were randomly sampled and analysed in detail. These form the denominator for this analysis. The primary diagnosis was gallstones in $62.8 \%$, malignancy in $16.9 \%$ and stricture in $10.2 \%$. Combinations of these or other diagnoses occurred in $17.6 \% .74 .5 \%$ of first ERCPs were urgent or emergent. Cannulation was attempted in $96.3 \%$ and successful in $81.5 \%$ of patients. The operator deemed the first ERCP to be successful in $40.6 \%$. Multiple stones requiring a stent and planned revisit occurred in $15.2 \%$ and a large stone requiring lithotripsy in $9.8 \%$. Repeat ERCP was deemed successful by the operator in $65.2 \%$ of cases. $40.2 \%$ went on to subsequent ERCP attempts. $31.1 \%$ of patients having a second or subsequent ERCP ended up having surgery (open biliary exploration, biliary bypass and other operations). On logistic regression, a primary diagnosis of gallstones was associated with likelihood of endoscopic success (OR (95\% CI): 3.8 (1.2 to $12.3, p=0.027$ ). In those patients with a primary diagnosis of gallstones, younger patients (OR 1.07 (1.01 to 1.12 , $\mathrm{p}=0.012$ )) and those with sepsis at presentation (OR 5.3 (1.1 to $25.2, \mathrm{p}=0.038)$ ) were significantly more likely to require surgery. No other pattern was predictive of subsequent ERCP success after a first attempt.

Conclusion From this analysis, there are no unequivocal clinical or technical factors which make either ERCP or surgery preferable following an incomplete first ERCP. Repeat ERCP should be considered in gallstone disease. In gallstone disease, younger or septic patients should be considered for early surgery if a first ERCP is not successful. This decision is not straightforward; multidisciplinary teamwork and communication between surgeon and endoscopist are essential.

Competing interests None declared.

\section{PTU-077 UNPLANNED HOSPITAL READMISSION WITHIN 30 DAYS AFTER LIVER AND PANCREATIC RESECTION}

doi:10.1136/gutjnl-2012-302514c.77

E L Neo, E Viñuela, * S R Bramhall, J Isaac, R Marudanayagam, D Mayer, D F Mirza, P Muiesan, R P Sutcliffe. Liver Unit, Queen Elizabeth Hospital, Birmingham, UK

Introduction Due to limited resources within the NHS, clinicians in the UK are under constant pressure to discharge patients rapidly, even after major surgery. There is a concern that premature discharge may lead to high readmission rates and worsen clinical outcomes. The aim of this study was to evaluate the incidence and outcome of unplanned hospital readmission after liver and pancreatic resection.

Methods Patients who underwent liver or pancreatic resection between January and December 2010 were identified from a prospective database. Potential risk factors for unplanned readmission within 30 days of discharge from hospital were evaluated. Complications (Clavien grade) and 90-day mortality were also assessed.

Results The median lengths of hospital stay after liver and pancreatic resections were 6 (range 4-66) and 9 days (range 5-225), respectively. 14/174 (8\%) patients were readmitted after hepatic resection. Type of liver resection was significantly associated with readmission (major $12.5 \%$ vs minor $3 \%$; $=0.03$ ). Of the readmitted patients, 7 (50\%) had grade 3 complications, including four patients who had an uncomplicated index admission, and two patients died due to sepsis. $10 / 100(10 \%)$ patients were readmitted after pancreatic resection. Readmission was more likely in patients with a pancreatic fistula $(30 \%$ vs $8 \%, p=0.06)$ and a white cell count $>16 \times 10^{9} / \mathrm{L}$ at the time of discharge $(50 \%$ vs $6 \%, p<0.001)$. Of the readmitted patients, 4 (40\%) had grade 3 complications, including 